\title{
Knockdown of tripartite motif-59 inhibits the malignant processes in human colorectal cancer cells
}

\author{
WEI WU ${ }^{1 *}$, JINGDI CHEN $^{2 *}$, JICHENG WU $^{3 *}$, JUN LIN $^{4}$, SHENG YANG $^{4}$ and HONGGANG YU ${ }^{1}$ \\ ${ }^{1}$ Department of Gastroenterology, Renmin Hospital of Wuhan University, Wuhan, Hubei 430060; ${ }^{2} 73$ th Contingent, \\ 95969 Troops, The Airborne Force of Chinese PLA, Wuhan, Hubei 430300; ${ }^{3}$ Tumor Basic and Translational Laboratory, \\ The First Affiliated Hospital of Jinzhou Medical University, Jinzhou, Liaoning 121000; ${ }^{4}$ Department of Gastroenterology, \\ Zhongnan Hospital of Wuhan University, Wuhan, Hubei 430071, P.R. China
}

Received March 3, 2017; Accepted August 1, 2017

DOI: $10.3892 /$ or.2017.5896

\begin{abstract}
The aim of the present study was to clarify the clinical implication and functional role of tripartite motif-59 (TRIM59) in colorectal carcinoma (CRC) and explore the underlying mechanism of aberrant high expression of TRIM59 in cancer. We validated that TRIM59 was upregulated in CRC samples, and also demonstrated that its upregulation was associated with advanced tumor stage of CRC patients; and its high expression indicated shorter overall survival and faster recurrence. Knockdown of TRIM59 significantly inhibited cell proliferation, migration and invasion. Cell cycle analysis showed that TRIM59-depleted cells accumulated in S-phase. In addition, the cell cycle regulators CDC25C, cyclin B1 and cyclin D1 were decreased by TRIM59 siRNA mediated knockdown. Furthermore, the depletion of TRIM59 promoted apoptosis in cell culture as indicated by the cleavage of caspase-3 and PARP when TRIM59 was depleted. These results suggested that TRIM59 is upregulated in human colorectal tumors compared with non-tumor tissues. The level of TRIM59 is correlated with malignant features of CRC and may serve as potential therapeutic and preventive strategies for CRC.
\end{abstract}

\section{Introduction}

Colorectal cancer (CRC) is one of the most commonly diagnosed cancer and one of the leading causes of cancer mortality worldwide (1). Metastasis accounts for $\sim 90 \%$ of patient deaths and is the most significant contributor to cancer death $(2,3)$. $\mathrm{CRC}$ is often diagnosed in its advanced stage accompanied

Correspondence to: Dr Honggang Yu, Department of Gastroenterology, Renmin Hospital of Wuhan University, 238 Jiefang Road, Wuhan, Hubei 430060, P.R. China

E-mail: yuhonggang1968@126.com

${ }^{*}$ Contributed equally

Key words: TRIM59, colorectal carcinoma, siRNA, proliferation, progression by metastasis, which is negatively correlated with patient survival (4). Since advanced metastatic CRC remains largely incurable, it is urgent to discover novel biomarkers which can influence its progression and contribute to diagnosis and treatment in early stage.

The tripartite motif (TRIM) proteins represent a large protein family comprising $>70$ members (5), constituted with a common N-terminal really interesting new gene (RING) finger domain, 1 or 2 B-box motifs and coiled-coil sequences, and most of them functioned as E3 ubiquitin ligases (6). According to previous studies, TRIM is implicated in many critical processes, such as viral infections, developmental and neurodegenerative disorders and cancer $(5,7)$. In recent years, many scholars have found that TRIM proteins act as oncogenes or tumor suppressors in cancer growth. For example, TRIM13, TRIM19, TRIM24 and TRIM25 have been linked to leukemia, breast and prostate cancer (8), respectively. TRIM59, through the regulation of transcriptional factors or tumor suppressors, is strongly associated with gastric tumor (9), NSCLC (10) and renal cell cancer (11), but the function of the TRIM59 in CRC has not yet been reported.

In this study, we analyzed TRIM59 from independent Gene Expression Omnibus (GEO) datasets (www.ncbi.nlm.nih.gov/ gds/) to determine the characteristic expression of TRIM59 in CRC. We found that TRIM59 is upregulated in CRC and significantly shortens patient survival through regulating cell proliferation, motility, cell cycle and inhibiting apoptosis of CRC cells.

\section{Materials and methods}

Patients and immunohistochemistry. Institutional Review Board (IRB)/Ethics Committee approval was obtained and the study was in adherence with the Declaration of Helsinki. The CRC samples and paired non-neoplastic tissues were obtained from Renmin Hospital of Wuhan University, and the study was approved by the local ethics committee. Before use, all cases were diagnosed by two pathologists without discrepancy. Formalin-fixed, paraffin-embedded CRC and non-neoplastic epithelial tissues were cut into $4 \mu \mathrm{m}$ sections. The sections were deparaffinized in xylene and rehydrated in a series of ethanol of descending concentrations. For antigen unmasking, sections 
were immersed in antigen-unmasking solution and boiled in a microwave oven for $15 \mathrm{sec}$. Tissue sections were incubated with the TRIM59 primary antibody at room temperature for $60 \mathrm{~min}$, followed by the standard procedure for the S-P immunohistochemical kit (Fujian Maixin Biological Technology, Co., Ltd., Fuzhou, China). Expression level of TRIM59 was ascertained according to the evaluations of the pathologists. The data of mRNA expression profile GSE14333 (12), GSE17536 (13), GSE4107 (14) and GSE32323 (15) were downloaded from the GEO database.

Cell culture, small interfering RNA and antibodies. HCT116, SW480, SW620, HT29, Caco2 and NCM460 cells were maintained in PRMI-1640 medium (Invitrogen) supplemented with $10 \%$ fetal bovine serum (FBS), $100 \mathrm{U} / \mathrm{ml}$ penicillin and $100 \mathrm{mg} / \mathrm{ml}$ streptomycin. Cells were cultured at $37^{\circ} \mathrm{C}$ in $5 \%$ $\mathrm{CO}_{2}$. Two different small interfering RNA (siRNA) oligo duplexes were produced by Invitrogen (Carlsbad, CA, USA). The target sequences for the TRIM59 gene were: siRNA-1: GCCUCUCUAUCUGUUUACCAAAGUU; siRNA-2: UCC UCGUGUACUGCCAUGCUCUCAU; The negative control from Invitrogen was used as a control. The specific siRNA or negative control was transfected into CRC cells with Lipofectamine 2000 transfection reagent (Invitrogen) according to the manufacturer's instructions. Primary antibody TRIM59 was purchased from Abcam (ab69639) and anti-caspase-3 (25546-1-AP), PARP (13371-1-AP), cyclin B1 (55004-1-AP), cyclin D1 (0186-1-Ig), CDC25C (16485-1-AP), MMP2 (103732-AP) were purchased from Proteintech (Rosemont, IL, USA).

Western blot analysis. CRC proteins were separated by SDS-PAGE. Proteins were electro-blotted onto a polyvinylidene fluoride membrane and were blocked for $30 \mathrm{~min}$ in TBST [0.05\% Tween-20 in phosphate buffered saline (PBS)] with 5\% dried skimmed milk at room temperature. Immunoblot analysis was performed using primary antibodies overnight at $4^{\circ} \mathrm{C}$. Membranes were then washed with TBST and incubated with horseradish peroxidase (HRP)-labeled secondary antibody in TBST for $45 \mathrm{~min}$ and then visualized using a chemiluminescent ECL detection kit (Santa Cruz Biotechnology, Santa Cruz, CA, USA). GAPDH was used as a control for protein loading.

RNA isolation and real-time PCR analysis. Total RNA was isolated from cell lines or tissues with TRIzol reagents (Invitrogen) according to the manufacturer's instructions. Quatitative real-time PCR (qRT-PCR) was performed to quantify mRNA expression with SYBR ${ }^{\circledR}$ Green PCR Master Mix (Takara Bio, Shiga, Japan). GAPDH was used for TRIM59 normalization. Primers were as follow: TRIM59: forward, 5'-TACGAGAGCAGCAGCTTGAA-3' and reverse, 5'-ACGG GTTGAACCTCAGGAAG-3'; GAPDH: forward, 5'-GTGGA CATCCGCAAAGAC-3' and reverse, 5'-AAAGGGTGTAAC GCAACTA-3'.

Cell proliferation assay. Cells were plated in 96-well plates and examined at 12, 24, 48 and $72 \mathrm{~h}$ after plating $(\mathrm{n}=6)$. Cells were incubated with the Cell Counting kit-8 (CCK-8; Dojindo Laboratories, Kumamoto, Japan) for $1 \mathrm{~h}$. Then the absorbance was measured with the multifunctional microplate reader at $450 \mathrm{~nm}$.
Cell cycle analysis. Cells were harvested and resuspended twice with PBS. After centrifugation, cells were fixed in $70 \%$ ice-cold methanol for at least $2 \mathrm{~h}$ at $-20^{\circ} \mathrm{C}$. Then the fixed cells were stained with propidium iodide (PI) $(50 \mu \mathrm{l} / \mathrm{ml} \mathrm{PI}$ and $250 \mu \mathrm{l} / \mathrm{ml}$ RNase A) for $30 \mathrm{~min}$. In each experiment, $2 \times 10^{5}$ cells were analyzed using BD FACSAria (BD Biosciences, San Jose, CA, USA). Experiments were performed in triplicate. ModFit 2.0 software was used to determine the percentage of cells in $\mathrm{G} 0 / \mathrm{G} 1, \mathrm{~S}$ and $\mathrm{G} 2 / \mathrm{M}$ phases.

Apoptosis analysis. Annexin V-FITC/PI staining was performed to investigate whether TRIM59 regulates the apoptosis of CRC cells. The cells of each group were stained with Annexin V-FITC/PI apoptosis detection kit (Invitrogen), according to the manufacturer's instructions. Experiments were performed in triplicate. FlowJo 7.6.1 software was used to determine the percentage of apoptotic cells.

In vitro migration and invasion assays. In the scratch wound healing assay, cells were cultured in serum-free medium for $24 \mathrm{~h}$ and wounded with pipette tips. Then the medium was refreshed. The wound closing procedure was observed $36 \mathrm{~h}$ later and images were taken. In vitro tumor cell migration was measured using Transwell chambers (Corning, Inc., Corning, NY, USA) according to the manufacturer's instruction. In brief, $2 \times 10^{5}$ cells with/without siRNA in $2 \%$ FBS RPMI-1640 medium were plated in the upper chamber and incubated with RPMI-1640 medium containing 10\% FBS in the bottom chamber for $48 \mathrm{~h}$. Subsequently, the number of cells on the bottom surface, which migrated across the membranes, were counted after staining by $0.1 \%$ crystal violet dye. Cell counting was carried out in five separate areas under a microscope. The invasion assay was the same except that Matrigel basement was contained in the BD BioCoat Matrigel Transwell Chambers (BD Biosciences, Bedford, MA, USA). The data are presented as mean \pm standard deviation (SD).

Statistical analysis. Survival analyses were carried out using the Kaplan-Meier method and the results were statistically analysed by the log-rank test. The correlations between the gene expression and the clinicopathological features were analyzed by the Chi-square test. A significant difference was defined as $\mathrm{P}<0.05$. Statistical analyses were performed using the GraphPad Prism (v.6.0).

\section{Results}

TRIM59 is upregulated in CRC on both $\mathrm{mRNA}$ and protein levels. To determine the significance of TRIM59 in CRC, we first analyzed the expression of TRIM59 at the mRNA level in the cohorts of CRC patients available from two independent GEO datasets GSE4107 and GSE32323. The two datasets involve paired CRC tumors and adjacent non-cancerous tissue mRNA information. We chose all the data of TRIM59 mRNA for statistical analyses. As shown in Fig. 1A, TRIM59 mRNA levels increased significantly in human colorectal tumor samples as compared with the adjacent normal colorectal tissues.

To verify the microarray analysis results, we performed immunoblot and qRT-PCR experiments on human colorectal 

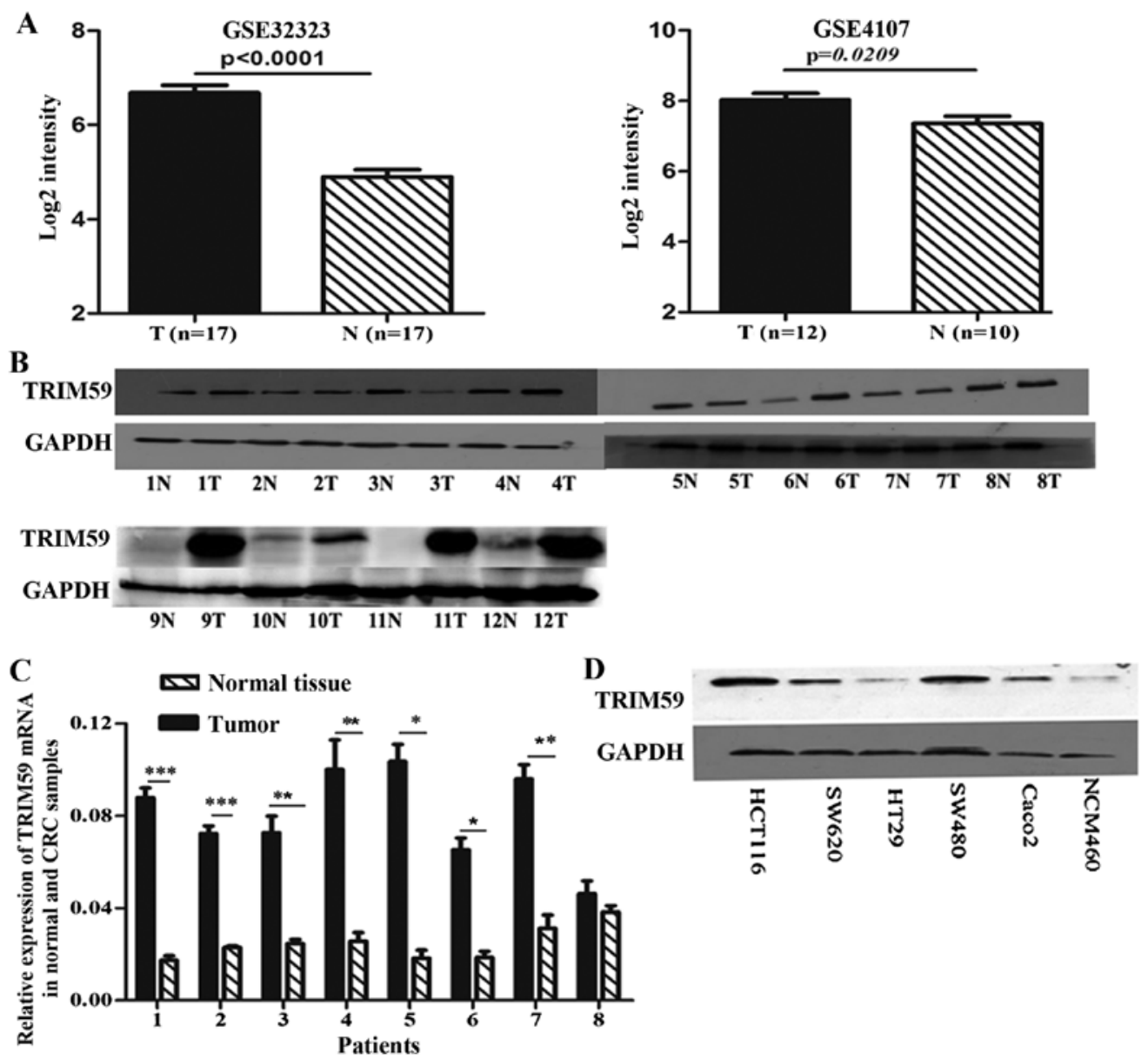

Figure 1. TRIM59 expression is upregulated in CRC. (A) TRIM59 expression level in CRC tissues and normal tissues in two independent cohorts (GSE32323 and GSE4107). (B) Immunoblot shows higher protein levels of TRIM59 in 7 of 12 tumor samples compared with the respective matched normal tissues (T, tumor; N, normal tissues). GAPDH is used as a loading control. (C) TRIM59 mRNA expression level in 8 paired tumor samples and normal tissues. (D) The expression of TRIM59 protein in five CRC cell lines and normal colon NCM460 cell line. ${ }^{*} \mathrm{P}<0.05,{ }^{* *} \mathrm{P}<0.01,{ }^{* * *} \mathrm{P}<0.001$.

adenocarcinoma specimens and their matched normal tissues. Seven of 12 tumor samples showed increased protein level of TRIM59 compared with their respective paired normal tissues (Fig. 1B). In addition, qRT-PCR experiments found significantly higher TRIM59 mRNA expression in the tumor tissues (Fig. 1C). Expression of TRIM59 was determined by western blot analysis in several CRC cell lines, including HCT116, SW480, SW620, HT29, Caco2 and the normal colon cell line NCM460. According to the tissue results, significantly higher expression of TRIM59 was detected in most CRC cells, compared to NCM460 cells (Fig. 1D). To further investigate the correlation of TRIM59 with CRC progression, we also examined the expression levels of TRIM59 in 80 human CRC tissues (60 cases of colon cancer and 20 cases of rectal carcinoma) and 16 normal colon tissues by immunohistochemical staining. TRIM59 was found mainly in the cytoplasm (Fig. 2A-E), which was consistent with previous reports $(9,16)$. The percentage of strongly positive samples in cancer tissues $(72.5 \%, 58 / 80)$ was significantly higher than that in normal colon tissues $(18.75 \%, 3 / 16)(\mathrm{P}<0.0001$, Yates' corrected $\chi^{2}$ analysis). Of the 60 colon cancer samples, 44 $(73.3 \%)$ were strongly positive and $16(26.7 \%)$ were weakly positive (Fig. 2A and B). Whereas, 14 (70\%) of the rectal cancer samples were strongly positive and only $6(30 \%)$ were weakly positive (Fig. 2C and D). In contrast, of the 16 normal tissue samples, 9 (56.25\%) were negative, 4 (25\%) were weakly positive and only 3 (27.9\%) were strongly positive (Fig. 2E and F). Distributions of TRIM59 staining grades are shown in Fig. 2G. Our results implied that TRIM59 expression was upregulated at the protein level in CRC.

These data evidently demonstrated that TRIM59 was upregulated in CRC in both mRNA and protein levels, implying its importance in CRC pathogenesis.

The expression level of TRIM59 is correlated with disease progression as well as shortened patient survival. In order to confirm the correlations between the TRIM59 expression level and the clinicopathological factors in CRC, we downloaded clinical information for GSE14333 and analyzed it statistically. The samples pooled in the dataset were then classified into two groups according to the TRIM59 expression level in tumor tissue and the $\chi^{2}$ test was applied. As shown (Table I), higher TRIM59 expression was closely associated with Dukes' stage $(\mathrm{P}=0.004)$. The results indicated that high expression of TRIM59 is related to rapid carcinoma spread. Moreover, we downloaded clinical information and performed Kaplan-Meier survival analysis. The two datasets (GSE17536 and GSE14333) contain mRNA information of CRC and disease-free survival 
A

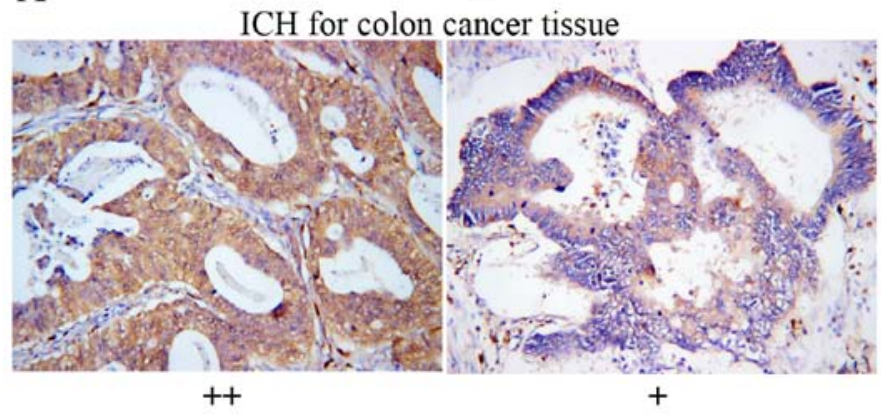

C

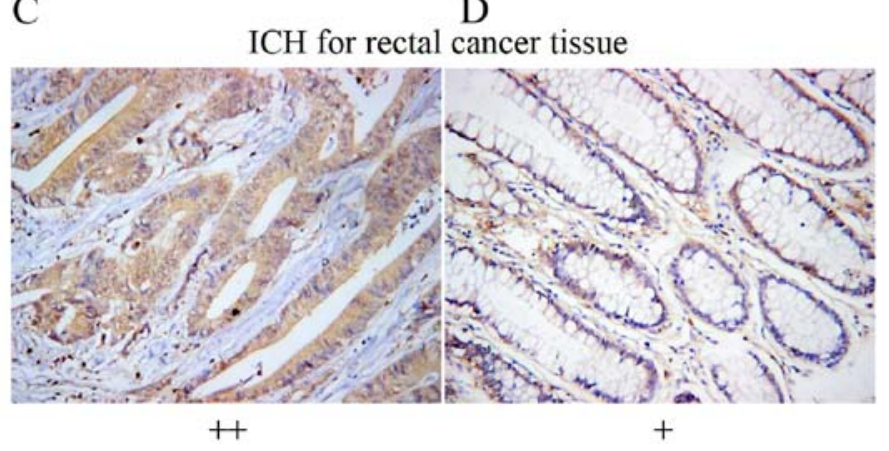

E

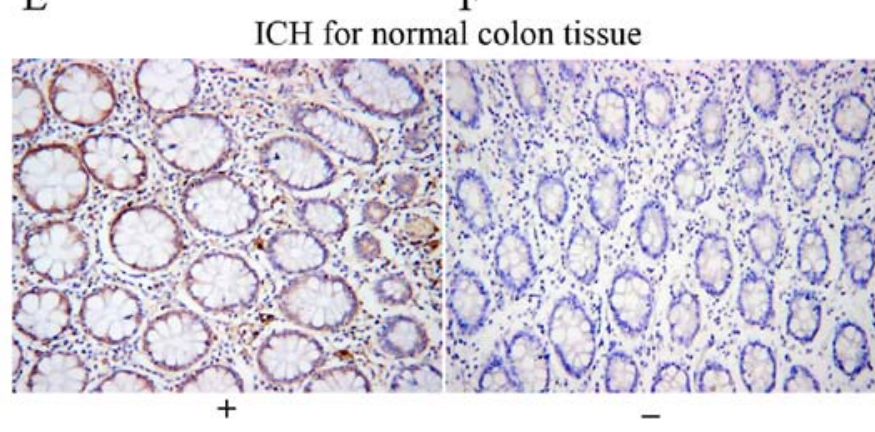

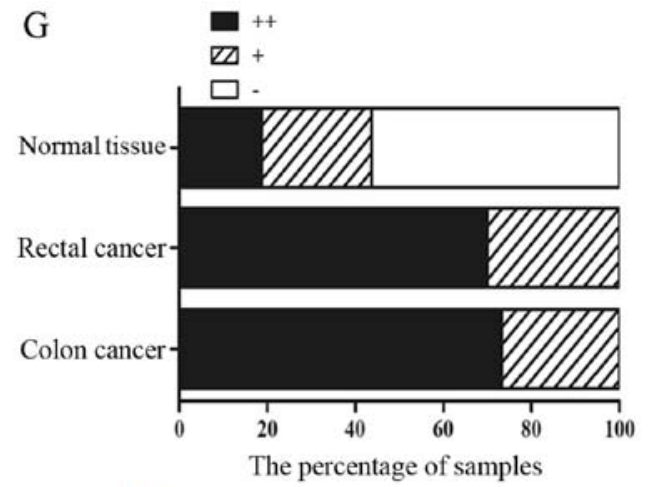
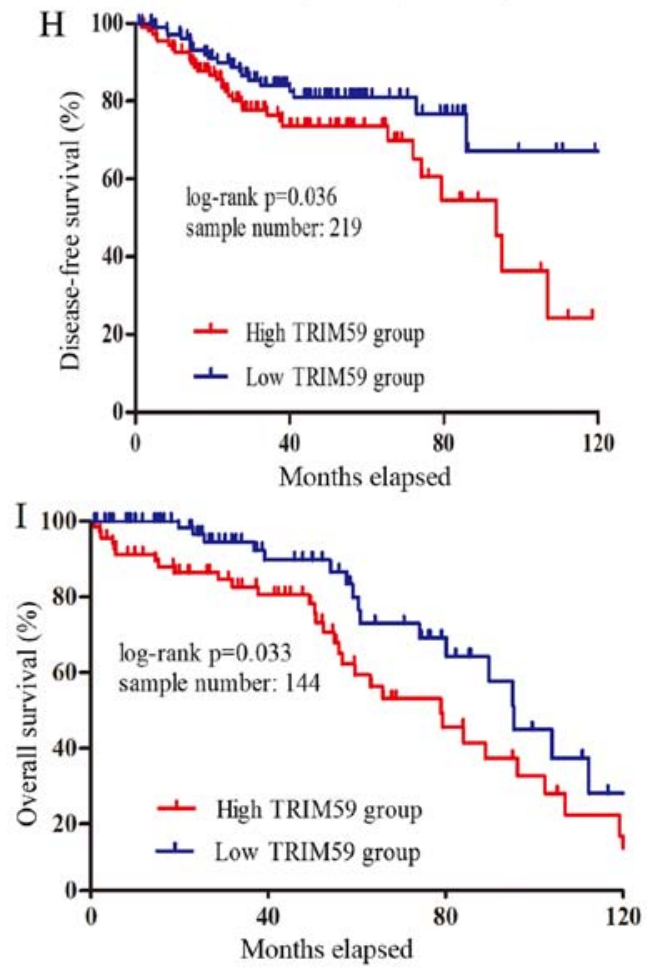

Figure 2. Increased expression of TRIM59 is correlated with poor survival in patients. (A-F) IHC staining of normal and CRC tissues. (A-D) Representative images of strongly positive staining $(++)$ and weakly positive staining $(+)$ of TRIM59 protein in colon cancer tissues (A and B) and rectal cancer tissues $(\mathrm{C}$ and D). (E and F) Representative imagess of negative staining (-) and weakly positive staining (+) of TRIM59 protein in normal colon tissues. (G) Distribution of TRIM59 staining grades $(-,+$ and ++) in normal colon tissues, colon cancer tissues and rectal cancer tissues. (H and I) Increased expression of TRIM59 is associated with a poor prognosis in CRC patients. (H) High TRIM59 mRNA level is associated with reduced OS in CRC patients in dataset GSE17536. (I) High TRIM59 mRNA level is associated with DFS in CRC patients in dataset GSE14333. IHC, immunohistochemical staining. Original magnification, $\mathrm{x} 200$.

(DFS) or overall survival (OS). We chose all the samples that contained complete TRIM59 mRNA and survival information for analysis. The results showed that CRC patients with tumors displaying high TRIM59 expression level had significantly shorter OS and DFS compared to those with tumors with low TRIM59 expression (Fig. $2 \mathrm{H}$ and $\mathrm{I})(\mathrm{P}=0.033$ and $\mathrm{P}=0.036$, respectively). The results strongly suggested that TRIM59 may act as an oncogene in CRC and could represent a new potential prognostic factor for CRC after curative colorectal resection.

TRIM59 modulates CRC cell proliferation. To validate the biological role of TRIM59 in the proliferation of CRC cells, TRIM59 was depleted using three siRNAs in HCT116 and SW480 cells, which exhibit a higher expression of TRIM59. After transfecting the three siRNAs into CRC cells, the protein level of TRIM59 was affirmed by western blot analysis. As shown in the Fig. 3A, both siRNA-1 and siRNA-2 efficiently knocked down TRIM59 in the two cell lines compared with that of control siRNA. Next, we examined the effects of siRNA-induced knockdown of TRIM59 on the growth of CRC cell lines. As shown in Fig. 3B, HCT116 and SW480 cells displayed a lower cell proliferation rate than control cells, there was statistical significant difference $(\mathrm{P}<0.05)$. These data suggested that TRIM59 is closely associated with the proliferation of CRC cells.

TRIM59 modulates cell migration and invasion of CRC cells. The role of TRIM59 in modulating CRC cell proliferation is in agreement with previous reports in cervical (17) and prostate cancer (16). In addition to cell proliferation, the role of TRIM59 in cancer metastasis has not been well characterized. We examined whether TRIM59 have an impact on cell migration and invasion. As shown in Fig. 4A and B, we performed a wound healing assay. The 'wound' was almost healed after 

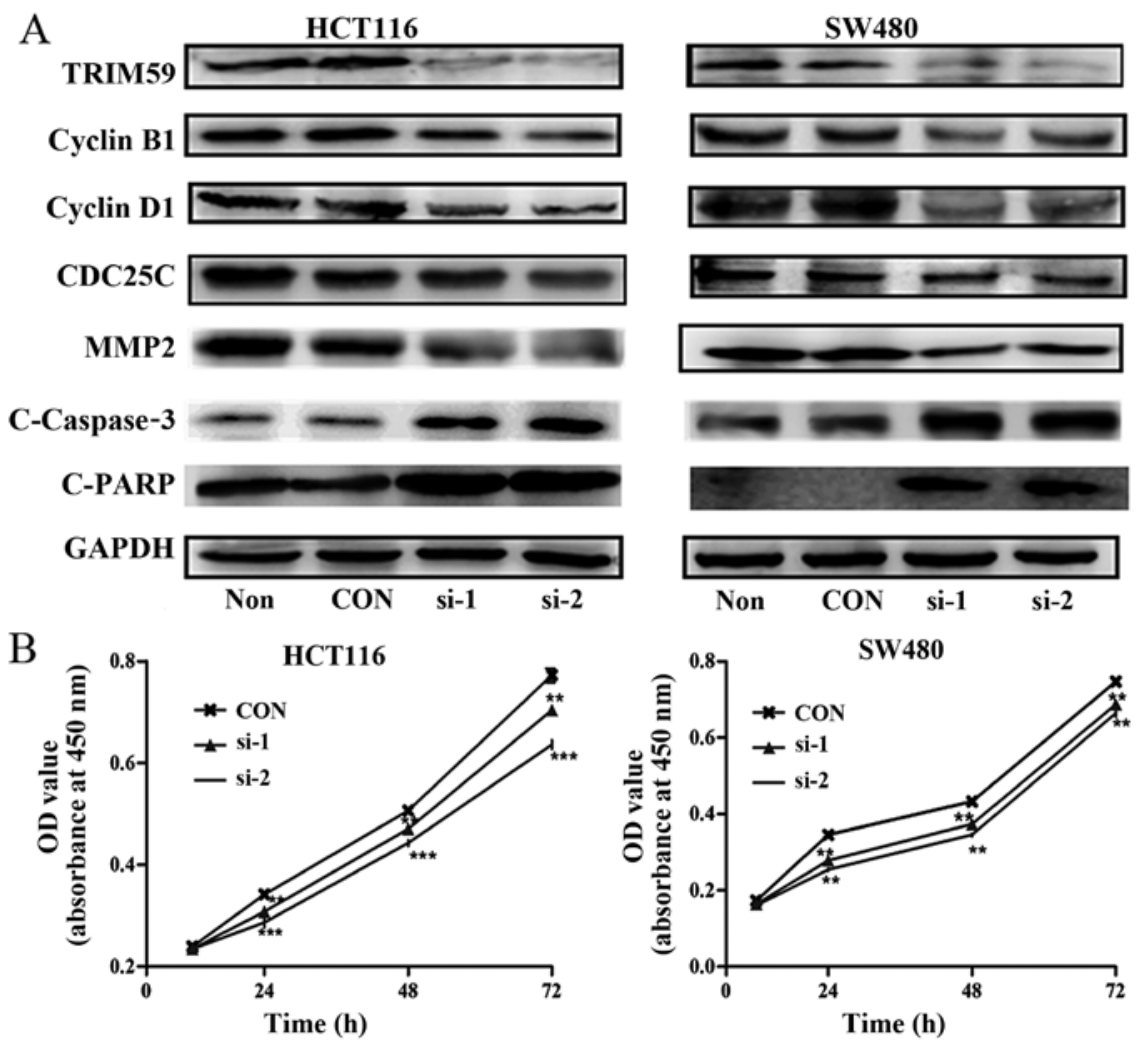

Figure 3. Knockdown of TRIM59 inhibits cell proliferation in CRC. (A) Knockdown of TRIM59 in HCT116 and SW480 by siRNA was confirmed by western blotting. GAPDH was used as a loading control. The protein expression levels of cyclin D1, cyclin B1, CDC25C, MMP2, C-caspase-3 and c-PARP were also checked by western blot analysis with indicated antibodies. (B) TRIM59 knockdown reduced the proliferation rate of HCT116 and SW480 cells. ** P $<0.01$, ${ }^{* * * *} \mathrm{P}<0.001$

Table I. Correlations between the TRIM59 expression and the clinicopathological features of colorectal carcinoma (GSE14333).

\begin{tabular}{|c|c|c|c|c|c|}
\hline \multirow[b]{2}{*}{ Characteristics } & \multirow{2}{*}{$\begin{array}{l}\text { No. of } \\
\text { patients }\end{array}$} & \multicolumn{2}{|c|}{$\begin{array}{l}\text { TRIM59 } \\
\text { expression }\end{array}$} & \multirow{2}{*}{$\begin{array}{l}\text { Chi-square } \\
\text { value }\end{array}$} & \multirow[b]{2}{*}{ P-value } \\
\hline & & High & Low & & \\
\hline Age (years) & & & & 0.258 & 0.612 \\
\hline$>60$ & 200 & 98 & 102 & & \\
\hline$\leq 60$ & 90 & 47 & 43 & & \\
\hline Sex & & & & 3.593 & 0.058 \\
\hline Male & 164 & 74 & 90 & & \\
\hline Female & 126 & 71 & 55 & & \\
\hline Location & & & & 1.466 & 0.48 \\
\hline L-colon & 138 & 64 & 74 & & \\
\hline R-colon & 111 & 60 & 51 & & \\
\hline Rectum & 39 & 19 & 20 & & \\
\hline Dukes' stage & & & & 13.067 & 0.004 \\
\hline A & 44 & 18 & 26 & & \\
\hline B & 94 & 40 & 54 & & \\
\hline $\mathrm{C}$ & 91 & 51 & 40 & & \\
\hline $\mathrm{D}$ & 61 & 42 & 19 & & \\
\hline
\end{tabular}

Data are presented as a number. L-colon, left half colon; R-colon, right half colon.
$36 \mathrm{~h}$ in control cells. However, the healing of the open area was markedly attenuated when TRIM59 was knocked down. In order to further prove this effect, we also performed a Transwell assay. Importantly, knockdown of TRIM59 suppressed the migration and invasion rates of HCT116 and SW480 cells (Fig. 4C and D). It is well known that MMPs, especially MMP-2, is significantly involved in the invasion and metastasis of human tumors (18). When TRIM59 is knocked down in HCT116 and SW480 cells, MMP2 is downregulated (Fig. 3A). TRIM59 modulates CRC cell migration and invasion. This may be by regulating the expression of MMP-2. These observations suggest that knockdown of TRIM59 significantly inhibits cell migration and invasion abilities in HCT116 and SW480 cells and upregulation of TRIM59 may have important consequences on the metastasis of CRC.

Knockdown of TRIM59 arrests cell cycle in S phase. Cell proliferation depends largely on cell cycle progression. Hence, the effect of TRIM59 knockdown on cell cycle progression was also assessed using flow cytometry. After treatment with si-TRIM59 or control siRNA for $48 \mathrm{~h}$, cells were collected and stained by PI. TRIM59 knockdown led to a significant accumulation of cells at the S-phase with cell proportion in G2/M phase largely decrease in HCT116 and SW480 cells (Fig. 5A). These data suggest that knockdown of TRIM59 interrupts cell cycle progression leading to the accumulation of cells in $\mathrm{S}$ phase. We proceeded to detect the expression levels of cyclins, which are key cell cycle regulators (19). As shown in Fig. 3A, the cyclin family proteins cyclin D1, CDC25C and cyclin B1 
A

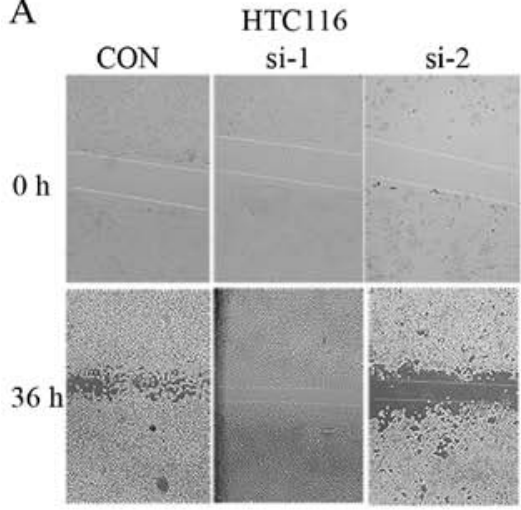

C

CON
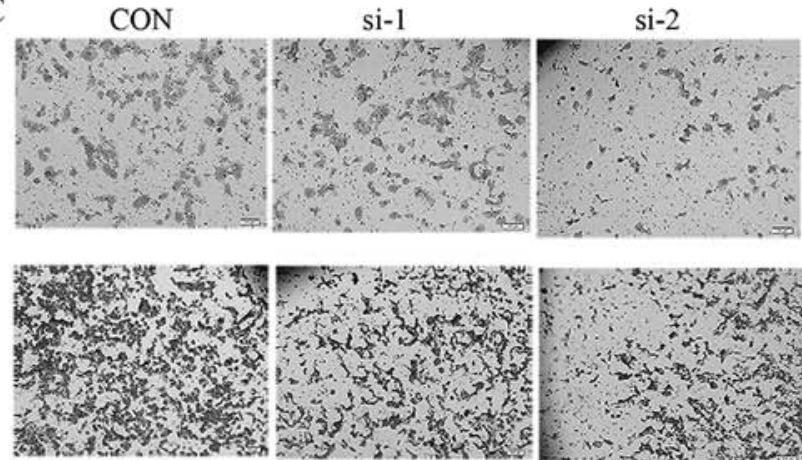

D

$\mathrm{CON}$
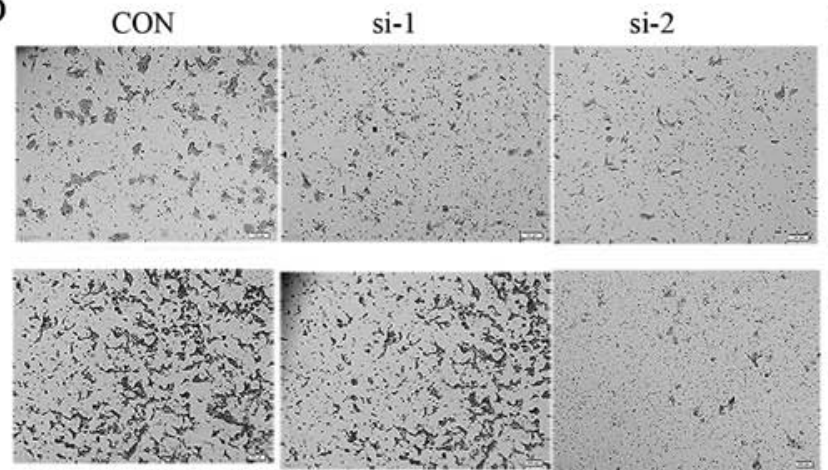

B
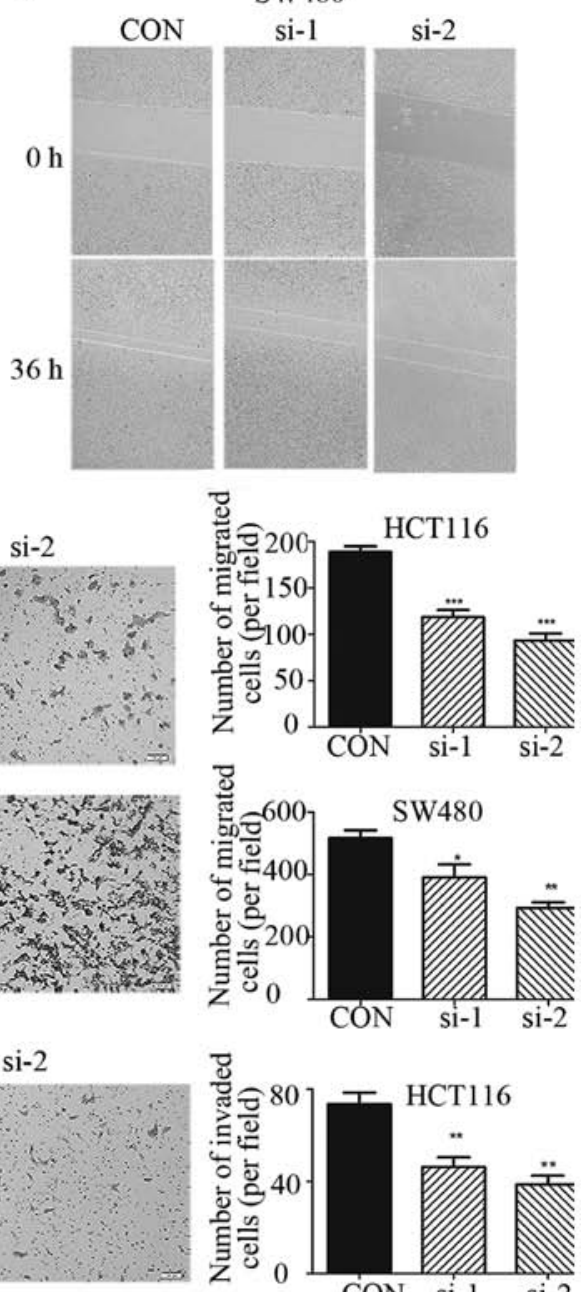

CON si-1 si-2

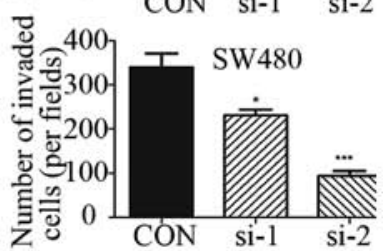

Figure 4. TRIM59 modulates migration and invasion ability in CRC cells. (A and B) Cell migration was assessed by scratch wound healing assay in HCT116 and SW480 cells. (C) Cell migration was assessed by Transwell migration assay in HCT116 (upper) and SW480 (bottom) cells after the cells were transfected with TRIM59 siRNA for $48 \mathrm{~h}$. (D) Cell invasion was assessed by Transwell invasion assay in HCT116 (upper) and SW480 (bottom) cells after the cells were transfected with TRIM59 siRNA for $48 \mathrm{~h}$. The bars represent the mean values of six independent tests $(\mathrm{mean} \pm \mathrm{SD}){ }^{*} \mathrm{P}<0.05,{ }^{* *} \mathrm{P}<0.01,{ }^{* * *} \mathrm{P}<0.001$.

were downregulated following TRIM59 knockdown, while the level of the internal control GAPDH remained stable. The regulation of cyclins by TRIM59 might underlie the TRIM59mediated cell cycle arrest in CRC cells.

Downregulation of TRIM59 causes apoptosis in CRC cells. Inhibiting apoptosis is crucial for sustaining proliferation. To further explore how TRIM59 regulates the proliferation of CRC cells, we investigated the contribution of apoptosis to growth inhibition in SW480 and HCT116 cells with or without TRIM59 knockdown. As expected, the apoptotic indexes of TRIM59-silenced cells and control cells were $7.19 \%$ (si-1), $13.37 \%$ (si-2) and $2.76 \%$ (control), (HCT116, P<0.05), $10.39 \%$ (si-1), $17.25 \%$ (si-2) and $4.66 \%$ (control) (SW480, P<0.05) (Fig. 5B), suggesting that silencing TRIM59 significantly promoted cell apoptosis.
Cell apoptosis is closely related to the regulation of apoptosis-related genes including Bcl-2 and caspase gene families (20). We measured apoptotic markers and the cleavage of caspase-3 and PARP (indicating a pro-apoptotic phenotype). These were higher when TRIM59 was depleted (Fig. 3A).

These results revealed that TRIM59 may exert an inhibitory effect on the caspase-dependent apoptosis pathway.

\section{Discussion}

The present study indicates that TRIM59 promotes CRC progression, which is similar to previous studies in other cancer types such as NSCLC (10), gastric cancer $(9,21)$, renal cell carcinoma (11) and osteosarcoma (22). Significantly shortened survival is seen in patients with high TRIM59 expression compared with those with low TRIM59 expression. Our data 
A
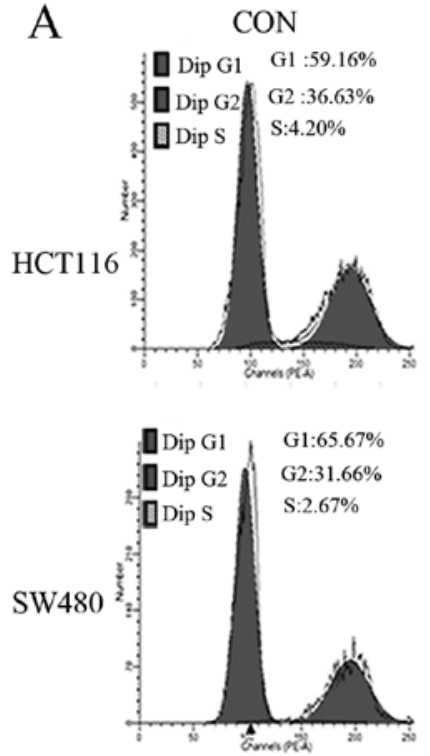

B

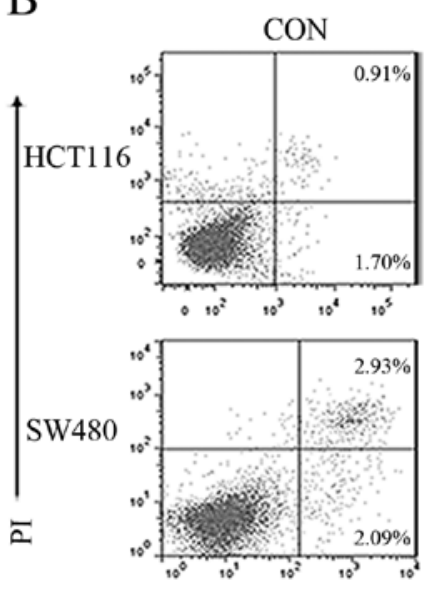

Annexin V-FITC
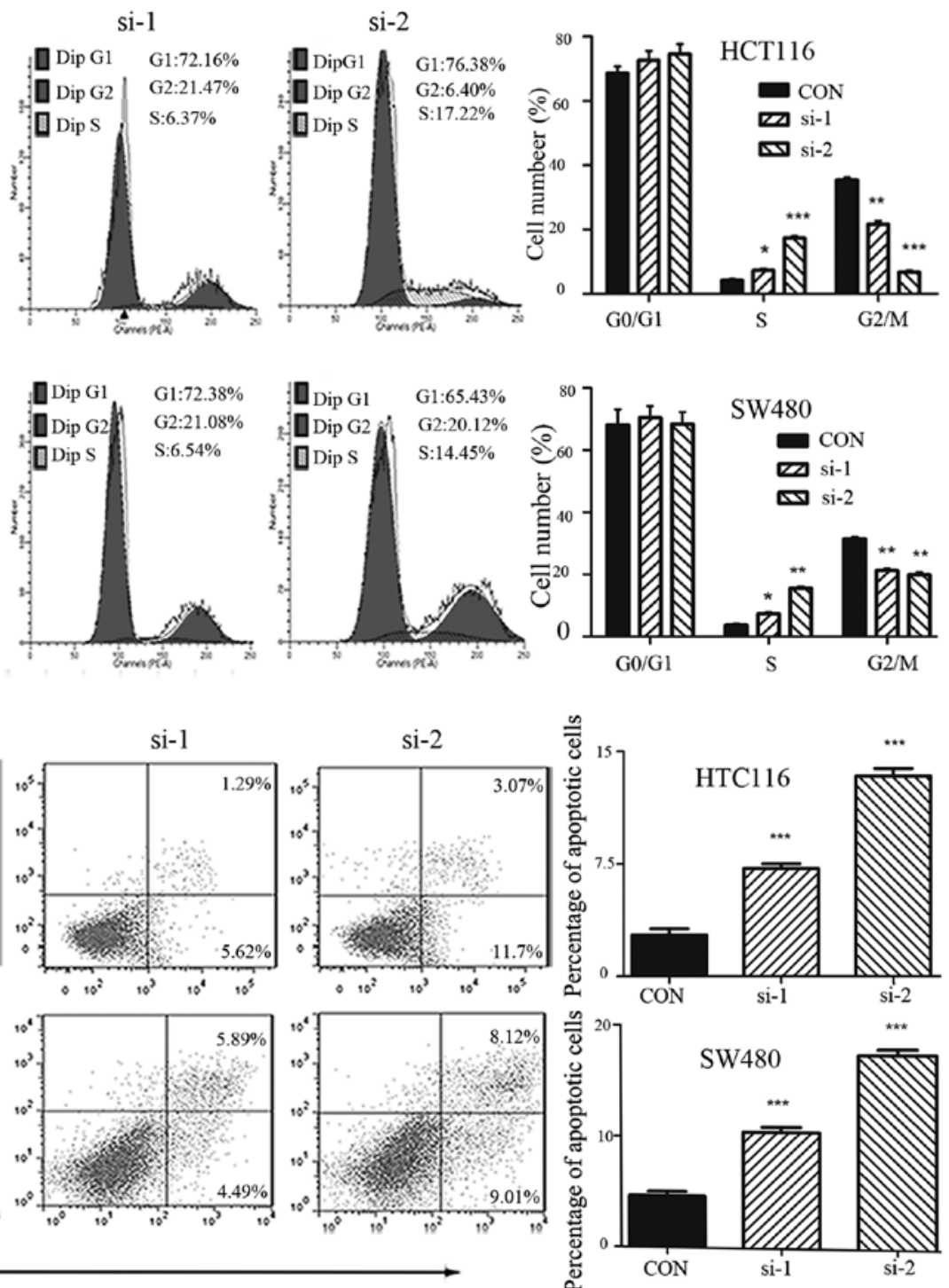

Figure 5. Knockdown of TRIM59 arrests cell cycle in S phase and promotes apoptosis. (A) The effects of knockdown of TRIM59 on the cell cycle. The percentage of cells in the G1, S and G2/M phases of the cell cycle is presented. (B) Apoptosis was determined by Annexin V-FITC and PI staining using flow cytometry. Representative apoptosis pattern is shown and the apoptotic cells are indicated in the UR and LR quadrants. ${ }^{*} \mathrm{P}<0.05,{ }^{* *} \mathrm{P}<0.01,{ }^{* * *} \mathrm{P}<0.001$.

showed knockdown of TRIM59 inhibited the cell cycle and promoted apoptosis in CRC cells in vitro, which may result in slower cancer cell proliferation. However, the underlying mechanism still remains unclear, and here several possible explanations are proposed.

Firstly, a hallmark of cancer cells is sustaining proliferative signaling, this can be achieved by inactivation of tumor suppressor genes. p53, a widely known tumor suppressor (23). At present, whether $\mathrm{p} 53$ is regulated by TRIM59 remain controversial. Some authors hold that p53 is negatively regulated by TRIM59 via ubiquinitation, by which TRIM59 promotes tumor growth, cell proliferation and migration (9). Hence, the SV40 Tag/p53/pRB routes can be considered one mechanism for TRIM59 functioning in the process of tumorigenesis. However, others believed that TRIM59 may promote cancer cell growth through other pathways but not the p53 signaling pathway (10). In this study, HCT116 is a p53 wild-type; in contrast, SW480 is mutant p53 cell line. When knocking down TRIM59, the two different cells have similar response, inferring that possibly TRIM59 is not mediated through p53, so further research is needed. In addition, the TRIM family is an evolutionarily conserved gene family implicated in a number of critical processes including transcriptional regulation $(24,25)$. The domain of the really interesting new gene (RING) is frequently involved in proteolysis, acting as E3 ubiquitin ligase and the ubiquitin-proteasome system in the regulation of numerous cellular processes including cell cycle regulatory proteins, transcription factors and signal transducers (26). Valiyeva et al (27) recently identified TRIM59 as an early signal transducer in two (SV40Tag and Ras) oncogene pathways in murine prostate cancer models. High expression level of TRIM59 may activate the transcription of some crucial downstream oncogenes, which, in turn promotes the progression of cancers. Furthermore, it is reported that TRIM59 is involved in NF- $\mathrm{KB}$ and IRF3/IRF7 mediated signaling pathways (28), both of which are dysregulated in nearly all tumors and considered to be driver genes of cancer. We hypothesize that high expression of TRIM59 will regulate NF- $\kappa B$ and 
IRF3/IRF7 pathways in CRC, which remains to be validated. Lastly, c-Myc overexpression and DNA promoter hypermethylation repress the expression of TRIM59 in cancers (29). These reports suggest several possible mechanisms by which TRIM59 may regulate cell cycle progression in colorectal cancer.

In the present study, we demonstrated that TRIM59 modulates not only proliferation, but also motility, migration and invasion. In CRC, metastasis accounts for $\sim 90 \%$ of patient deaths, representing the most lethal event during the course of the disease (30). Metastasis is directly linked to patient survival, and critically limits successful therapy $(31,32)$. Metastasis formation is a major hurdle in CRC therapy. Therefore, to identify patients at high risk for metastasis formation, early diagnosis and molecular characterization of the primary tumor is crucial to define prognostic and therapeutic targets (33). Our results indicated that TRIM59 is an important target for blocking metastasis in CRC. We propose that quantification of TRIM59 in colon biopsies could be used in combination with pathologic examination to predict biological behavior of the CRC. The molecular pathologic diagnosis will be helpful in personalized treatment optimization.

In summary, the present study has shown the biological and clinical significance of TRIM59 in CRC. This study demonstrated that TRIM59 expression is significantly upregulated in CRC tissues and associated with tumor Dukes' stage. Knockdown of TRIM59 with specific siRNA inhibited cancer cell proliferation and led to cell cycle arrest in the S-phase. Regulation of cell cycle progression and promotion of apoptosis may underlie TRIM59-mediated CRC development. Our evidence provides novel clues that may aid CRC diagnosis and treatment in the future. However, further in depth exploration of the molecular mechanisms of TRIM59 in promoting proliferation and metastasis will be needed.

\section{Acknowledgements}

The authors would like to thank the anonymous reviewers for their helpful comments. The authors also would like to thank Dr Katelyn O'Neill from the University of Nebraska Medical Center for improving the language of this manuscript.

\section{References}

1. Terzic J, Grivennikov S, Karin E and Karin M: Inflammation and colon cancer. Gastroenterology 138: 2101-2114.e5, 2010.

2. Stein U and Schlag PM: Clinical, biological, and molecular aspects of metastasis in colorectal cancer. Recent Results Cancer Res 176: 61-80, 2007.

3. Li W, Cai S, Wang L, Yang C, Zhou B and Wang H: HINT2 downregulation promotes colorectal carcinoma migration and metastasis. Oncotarget 8: 13521-13531, 2017.

4. Mathot L, Kundu S, Ljungström V, Svedlund J, Moens L, Adlerteg T, Falk-Sörqvist E, Rendo V, Bellomo C, Mayrhofer M, et al: Somatic ephrin receptor mutations are associated with metastasis in primary colorectal cancer. Cancer Res 77 : 1730-1740, 2017.

5. Elabd S, Meroni G and Blattner C: TRIMming p53's anticancer activity. Oncogene 35: 5577-5584, 2016.

6. Ozato K, Shin DM, Chang TH and Morse HC III: TRIM family proteins and their emerging roles in innate immunity. Nat Rev Immunol 8: 849-860, 2008.

7. Tomar D and Singh R: TRIM family proteins: Emerging class of RING E3 ligases as regulator of NF-אB pathway. Biol Cell 107: 22-40, 2015 .
8. Hatakeyama S: TRIM proteins and cancer. Nat Rev Cancer 11: 792-804, 2011.

9. Zhou Z, Ji Z, Wang Y, Li J, Cao H, Zhu HH and Gao WQ: TRIM59 is up-regulated in gastric tumors, promoting ubiquitination and degradation of p53. Gastroenterology 147: 1043-1054, 2014.

10. Zhan W, Han T, Zhang C, Xie C, Gan M, Deng K, Fu M and Wang JB: TRIM59 promotes the proliferation and migration of non-small cell lung cancer cells by upregulating cell cycle related proteins. PLoS One 10: e0142596, 2015.

11. Khatamianfar V, Valiyeva F, Rennie PS, Lu WY, Yang BB, Bauman GS, Moussa M and Xuan JW: TRIM59, a novel multiple cancer biomarker for immunohistochemical detection of tumorigenesis. BMJ Open 2: e001410, 2012.

12. Jorissen RN, Gibbs P, Christie M, Prakash S, Lipton L, Desai J, Kerr D, Aaltonen LA, Arango D, Kruhøffer M, et al: Metastasisassociated gene expression changes predict poor outcomes in patients with Dukes stage B and C colorectal cancer. Clin Cancer Res 15: 7642-7651, 2009.

13. Freeman TJ, Smith JJ, Chen X, Washington MK, Roland JT, Means AL, Eschrich SA, Yeatman TJ, Deane NG and Beauchamp RD: Smad4-mediated signaling inhibits intestinal neoplasia by inhibiting expression of $\beta$-catenin. Gastroenterology 142: 562-571.e2, 2012.

14. Hong Y, Ho KS, Eu KW and Cheah PY: A susceptibility gene set for early onset colorectal cancer that integrates diverse signaling pathways: Implication for tumorigenesis. Clin Cancer Res 13: $1107-1114,2007$.

15. Khamas A, Ishikawa T, Shimokawa K, Mogushi K, Iida S, Ishiguro M, Mizushima H, Tanaka H, Uetake H and Sugihara K: Screening for epigenetically masked genes in colorectal cancer Using 5-Aza-2'-deoxycytidine, microarray and gene expression profile. Cancer Genomics Proteomics 9: 67-75, 2012.

16. Lin WY, Wang H, Song X, Zhang SX, Zhou PS, Sun JM and Li JS: Knockdown of tripartite motif 59 (TRIM59) inhibits tumor growth in prostate cancer. Eur Rev Med Pharmacol Sci 20: 4864-4873, 2016.

17. Aierken G, Seyiti A, Alifu M and Kuerban G: Knockdown of tripartrtite-59 (TRIM59) inhibits cellular proliferation and migration in human cervical cancer cells. Oncol Res 25: 381-388, 2016.

18. Xing P, Liao Z, Ren Z, Zhao J, Song F, Wang G, Chen K and Yang $\mathrm{J}$ : Roles of low-density lipoprotein receptor-related protein 1 in tumors. Chin J Cancer 35: 6, 2016.

19. Koosha S, Alshawsh MA, Looi CY, Seyedan A and Mohamed Z: An association map on the effect of flavonoids on the signaling pathways in colorectal cancer. Int J Med Sci 13: 374-385, 2016.

20. Shu YJ, Weng H, Ye YY, Hu YP, Bao RF, Cao Y, Wang XA, Zhang F, Xiang SS, Li HF, et al: SPOCK1 as a potential cancer prognostic marker promotes the proliferation and metastasis of gallbladder cancer cells by activating the PI3K/AKT pathway. Mol Cancer 14: 12, 2015.

21. Luo D, Wang Y, Huan X, Huang C, Yang C, Fan H, Xu Z and Yang L: Identification of a synonymous variant in TRIM59 gene for gastric cancer risk in a Chinese population. Oncotarget 8: 11507-11516, 2016.

22. Liang J, Xing D, Li Z, Shen J, Zhao H and Li S: TRIM59 is upregulated and promotes cell proliferation and migration in human osteosarcoma. Mol Med Rep 13: 5200-5206, 2016.

23. Charni M, Aloni-Grinstein R, Molchadsky A and Rotter V: p53 on the crossroad between regeneration and cancer. Cell Death Differ 24: 8-14, 2017.

24. Gack MU, Shin YC, Joo CH, Urano T, Liang C, Sun L, Takeuchi O, Akira S, Chen Z, Inoue S, et al: TRIM25 RING-finger E3 ubiquitin ligase is essential for RIG-I-mediated antiviral activity. Nature 446: 916-920, 2007.

25. Lerner M, Corcoran M, Cepeda D, Nielsen ML, Zubarev R, Pontén F, Uhlén M, Hober S, Grandér D and Sangfelt O: The RBCC gene RFP2 (Leu5) encodes a novel transmembrane E3 ubiquitin ligase involved in ERAD. Mol Biol Cell 18: 1670-1682, 2007.

26. Deshaies RJ and Joazeiro CA: RING domain E3 ubiquitin ligases. Annu Rev Biochem 78: 399-434, 2009.

27. Valiyeva F, Jiang F, Elmaadawi A, Moussa M, Yee SP, Raptis L, Izawa JI, Yang BB, Greenberg NM, Wang F, et al: Characterization of the oncogenic activity of the novel TRIM59 gene in mouse cancer models. Mol Cancer Ther 10: 1229-1240, 2011. 
28. Kondo T, Watanabe M and Hatakeyama S: TRIM59 interacts with ECSIT and negatively regulates NF- $\mathrm{BB}$ and IRF-3/7mediated signal pathways. Biochem Biophys Res Commun 422: 501-507, 2012.

29. Licchesi JD, Van Neste L, Tiwari VK, Cope L, Lin X, Baylin SB and Herman JG: Transcriptional regulation of Wnt inhibitory factor-1 by Miz-1/c-Myc. Oncogene 29: 5923-5934, 2010.

30. Dahlmann M, Kobelt D, Walther W, Mudduluru G and Stein U: S100A4 in cancer metastasis: Wnt signaling-driven interventions for metastasis restriction. Cancers (Basel) 8: E59, 2016.

31. Fodde R, Smits R and Clevers H: APC, signal transduction and genetic instability in colorectal cancer. Nat Rev Cancer 1: 55-67, 2001.
32. Stein U and Schlag PM: Clinical, biological, and molecular aspects of metastasis in colorectal cancer. Recent Results Cancer Res 176: 61-80, 2007.

33. Dahlmann M, Okhrimenko A, Marcinkowski P, Osterland M, Herrmann P, Smith J, Heizmann CW, Schlag PM and Stein U: RAGE mediates S100A4-induced cell motility via MAPK/ERK and hypoxia signaling and is a prognostic biomarker for human colorectal cancer metastasis. Oncotarget 5: 3220-3233, 2014. 